\title{
DESIGNING PULSE POWER GENERATORS
}

By:

K.R. Davey

J.R. Uglum

Plasma Science, vol. 33, no. 4, August 2005, pp. 1170-1176

PR 399

Center for Electromechanics The University of Texas at Austin

PRC, Mail Code R7000

Austin, TX 78712

(512) $471-4496$

$01 / 01 / 2005$ 


\title{
Designing Pulse Power Generators
}

\author{
Kent Davey, Fellow IEEE and John Uglum
}

\begin{abstract}
When the performance criteria for a pulsed power generator is power density, and the duty cycle remains short $(<20$ seconds), then copper coils with an exciter are favored over permanent magnet rotors. If the permanent magnets are replaced with copper coils, steel, and an exciter, with the same total weight, the copper coil alternative will return a higher MMF/weight, and thus a higher power density system. A variable metric optimization is completed for a generator assuming the objective is to charge a capacitor bank. The equations governing allowed current density in capacitor charging applications and ac/dc resistance ratios are derived.
\end{abstract}

Keywords - permanent magnet, copper, adiabatic, power density, generator, ac - dc resistance

\section{INTRODUCTION}

High power devices present special problems for power generation. Flywheels and capacitor banks are common methods of storing the energy because both allow energy removal quickly. But energy storage is only half the problem. Repeatedly converting that energy to useful power presents new challenges to generator designers. Indeed their design is quite different from conventional generator design. Much of that has to do with the unique thermal problems presented with short duty cycle machines. The short duty cycle also presents interesting engineering trades in the choice of rotor field. This paper discusses some of the unique aspects of very high power, short duty power generation, and its implication in the design of electromechanical devices.

\section{REQUIREMENTS}

The performance metric for pulsed power generators is either power/volume or power/weight. Both performance standards drive the design in nearly the same direction. The following generator requirements are assumed in this discussion:

- Charge a capacitor to $60 \mathrm{MJ}$ in 9 seconds

- The generator takes its energy from a flywheel, either directly integrated into the generator or attached to the same shaft

- The depth of inertial energy discharge after $9 \mathrm{~s}$ is $65 \%$

- The duty cycle is 1 hour, i.e., one hour elapses before the next pulse power event occurs

\section{Permanent Magnets versus Copper Coils}

Manuscript submitted May 1, 2004. Kent Davey and John Uglum are research scientists with the Center for Electromechanics, University of Texas, 10100 Burnet Rd, EME 133, Austin, TX 78759 (phone: 512-232-1603, email k.davey@mail.utexas.edu). John Uglum ca be reached at 512-232-1691, email j.uglum@mail.utexas.edu.
Among the most important questions in weight sensitive applications is whether to use permanent magnets or copper coils for the field MMF. The question is not at all sophomoric. A cavalier assertion would be "Use permanent magnets because the weight of a field exciter is lost, and a magnet ring can be spun at a higher speed than a copper coils employing steel laminations." The perfunctory nature of this response ignores the following:

$>$ Extremely short duty cycles allow high current densities in copper

$>$ The back iron so critical to flux closure for copper circuits can also be treated as dead weight wrapped by carbon composite, in the same fashion as the magnets are treated.

$>$ New carbon composite materials are available which have a $27.7 \%$ iron cobalt content by volume. Such fibers are presently being manufactured for magnetic shielding applications, but never for something such as proposed here. These fibers have the potential to provide considerable assistance to both permanent magnet and copper coil topologies, but more so to copper coils.

$>$ Pulsed power generators operate at high speed and current. The heat produced by both degrades not only the performance of permanent magnet options, but also their life.

$>$ Permanent magnets suffer a performance degradation with temperature. Among the three source of heat in this application are the following:

\section{o Windage from high rotational speed}

o Power dissipation in the coils of the stator

o Eddy current dissipation within the magnet due by slot harmonics. Neodymium magnets have a conductivity of $6.944 \cdot 10^{5} \mathrm{~S} / \mathrm{m}$. Kawase [1][2] computes a $0.012 \%$ (by power) eddy loss within the permanent magnets of a 6 pole $500 \mathrm{~kW}$ synchronous machine operating at 1200 RPM using 36 slots. The loss is proportional to power rating and speed ${ }^{2}$. This would translate to $1.8 \%$ at 15,000 RPM.

$>$ Permanent magnet systems have hardware control restrictions related to inrush current that add weight to the system. (Copper coil systems have the added weight of an exciter).

\section{A. Physics governing copper coils in pulsed power applications}

The current density appropriate for copper coils is dictated by the short duty cycle. Its value is chosen so that the adiabatic jump in temperature during the charge cycle remains below 
that allowed by the insulation, which in this study is about $100^{\circ} \mathrm{C}$. To defend this properly, a brief tutorial on the capacitor charging applications is in order.

Capacitor charging is most efficient when the current into the capacitor is constant, one like that depicted in Fig. 1. Any other choice with the same average will always produce a higher $\int I^{2} R$ loss factor.

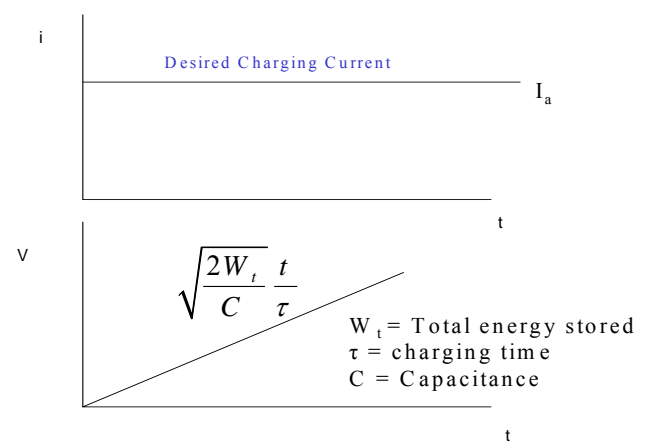

Fig. 1 Desired capacitor current and commensurate voltage.

The commensurate power and energy flow into the capacitor is shown in Fig. 2. If the energy is supplied by a flywheel, the flywheel speed will fall off quadratically as depicted in Fig. 3.

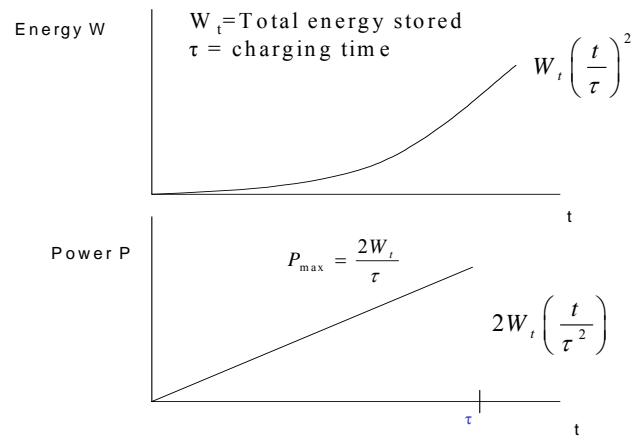

Fig. 2 Power and energy delivery to the capacitor.

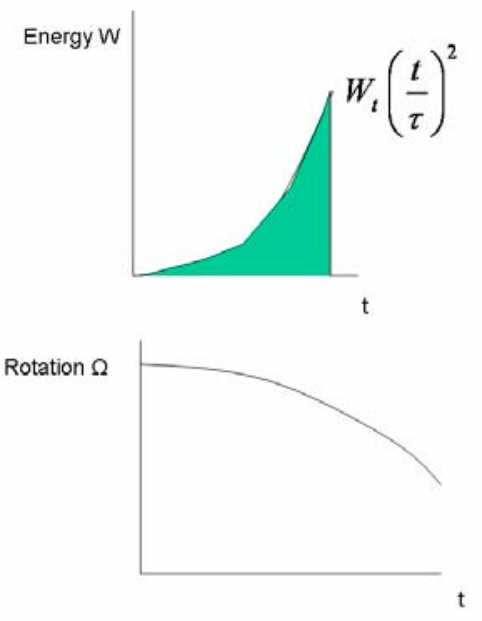

Fig. 3 Rotation of the flywheel with time.
Let $\tau$ represent the active excitation time, Im the combined mass inertia of the flywheel and the generator, and $\beta$ the allowed flywheel energy loss. Energy balance requires the flywheel speed with time to be

$$
\Omega=\sqrt{\Omega_{0}{ }^{2}-\frac{C V_{f}{ }^{2}}{I_{m}}\left(\frac{t}{\tau}\right)^{2}}=\Omega_{0} \sqrt{\left(1-\beta\left(\frac{t}{\tau}\right)^{2}\right)}
$$

$\Omega_{0}$ represents the initial mechanical speed. Since power is the product of torque and speed, the torque will have the time dependence

$$
T=\frac{I_{a} V_{f}\left(\frac{t}{\tau}\right)}{\Omega_{0} \sqrt{\left(1-\beta \cdot\left(\frac{t}{\tau}\right)^{2}\right)}}
$$

$I_{a}$ is the armature current, and $V_{f}$ the final armature voltage. This rapid rise is graphed in Fig. 4.

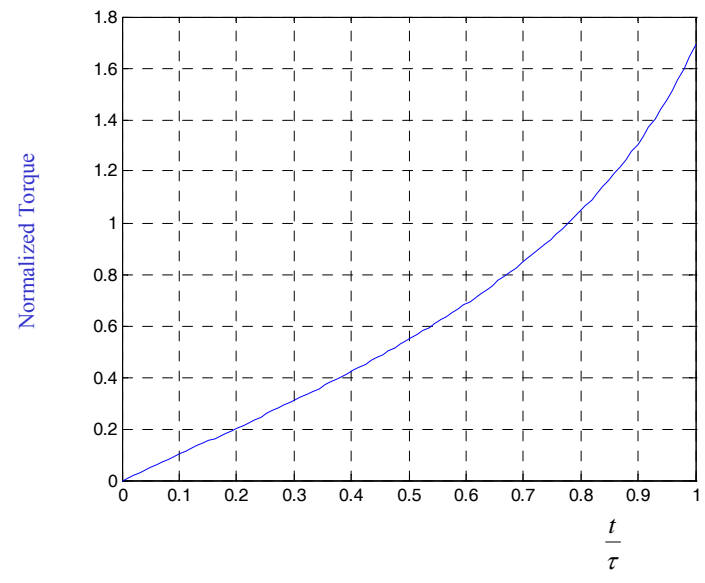

Fig. 4 Torque required on the charging generator.

\section{B. $\quad$ Upper Limits on Copper Current Density}

How hard can the copper coils in the generator be pushed in a pulsed power duty cycle? The constant current stator excitation is a bit easier to compute. Consider a one turn winding having cross sectional area $\mathrm{A}$, length $\mathrm{L}$, conductivity $\sigma$, mass density $\rho$, carrying current density J. The resistive dissipation in the winding is

$$
P=(J \cdot A)^{2} \frac{L}{\sigma A}
$$

Adiabatic heating demands a commensurate temperature change $\Delta \mathrm{T}$ in $\delta \mathrm{t}$ seconds of

$$
P \delta t=\rho C_{p}(A L) \Delta T
$$

Substituting (3) into (4) yields the current density in this one turn winding independent of area to be

$$
J=\sqrt{\frac{\rho \sigma C_{p} \cdot \Delta T}{\delta t}}
$$

Irrespective of the conductor size, the current density is dictated by the adiabatic temperature jump allowed. The conductivity, density, and specific heat for copper are 
respectively $5.810^{7} \mathrm{~S} / \mathrm{m}, 8.96 \cdot 10^{3} \mathrm{~kg} / \mathrm{m}^{3}\left(0.323 \mathrm{lbs} / \mathrm{in}^{3}\right)$, and $383 \mathrm{~J} / \mathrm{kg} / \mathrm{K}$. The conductivity drops to $4.310^{7} \mathrm{~S} / \mathrm{m}$ at $100^{\circ} \mathrm{C}$. The total loss in the stator slot is complicated by the slot induced eddy currents. Fig. 5 shows a geometry suitable for estimating that loss. The $\mathrm{x}$ directed $\mathrm{H}$ field in the slot is due to the number of conductors with current below it,

$$
H_{x}=\frac{n I}{a}
$$

Fig. 6 shows a blowup of the magnetic field density around an isolated slot with 60 sub-conductors. The horizontal slot is rotated $90^{\circ}$ from that depicted in Fig. 5. Note that even in an accurate numerical analysis how the primary component of the magnetic field remains straight across the slot, except in the vicinity of the air gap.

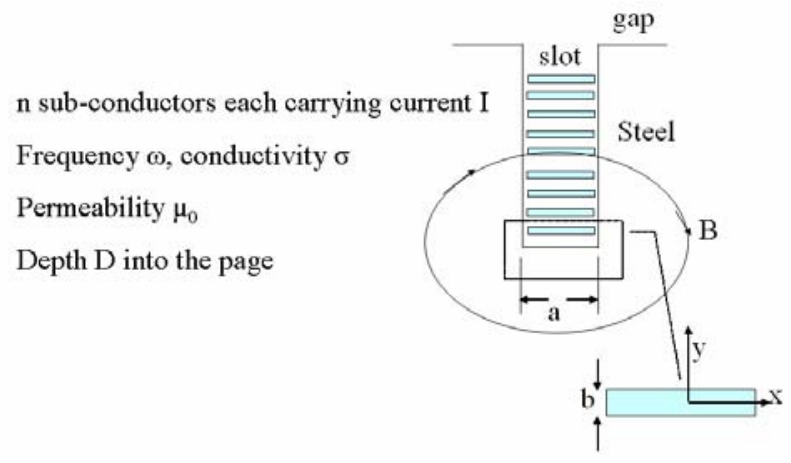

Fig. 5 Geometry used for computing excess conductor slot loss.

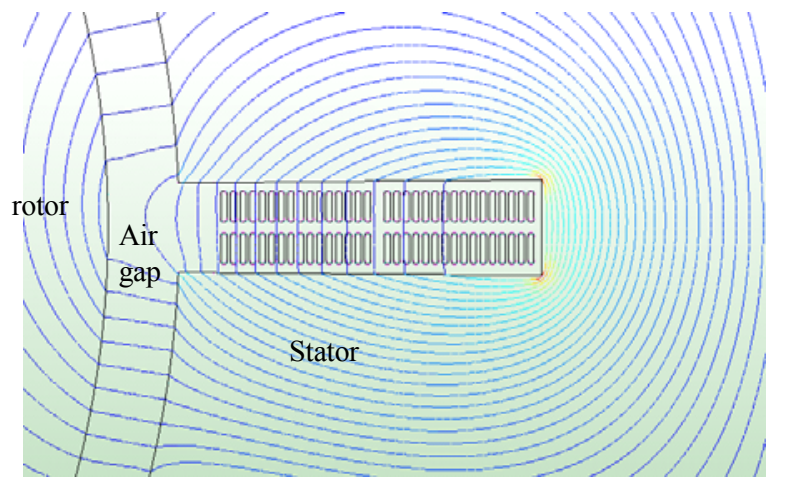

Fig. 6 Magnetic field density plot around an isolated stator slot with 60 sub-conductors. The slot is rotated $90^{\circ}$ from that depicted in Fig. 5.

Using Faraday's law at excitation frequency $\omega$, it follows that the $\mathrm{z}$ directed $\mathrm{E}$ field within a conductor at position $\mathrm{n}+1$, having $\mathrm{n}$ conductors below it is

$$
E_{z}=\frac{j \omega \mu_{0} n I}{a} y
$$

The real power dissipation per unit volume is $P_{v}=\Re\left\{\sigma E \cdot E^{*}\right\}$. Integrating this over the conductor thickness from $-b / 2$ to $b / 2$ gives the conductor loss

$$
P=D a \int_{-b / 2}^{b / 2} \sigma\left(\frac{\omega \mu_{0} n I}{a}\right)^{2} y^{2} d y=\left(\frac{D I^{2}}{\sigma a b}\right) \frac{\left(\omega \mu_{0} \sigma n b^{2}\right)^{2}}{12}
$$

Note that $\mathrm{n}$ represents the number of turns below the conductor in question. There is a small self field component which is ignored in the estimate; this ignored component is even less significant in view of the fact that the designer is most concerned about loss in the top of the slot which typically has multiple conductors below it. The multiplier $\left(\frac{D I^{2}}{\sigma a b}\right)$ is the dc loss within the conductor. Labeling this the $\mathrm{dc}$ loss $\mathrm{P}_{\mathrm{dc}}$, the total loss in the slot would be

$$
P_{\text {total @ position } n+1}=P_{d c}+P_{a c}=P_{d c}\left[1+\frac{\left(\omega \mu_{0} \sigma n b^{2}\right)^{2}}{12}\right]
$$

Typical loss values for a 32 conductor slot with $b=0.0702$ $\mathrm{cm}(0.0276 ")$ are shown in Fig. 7.

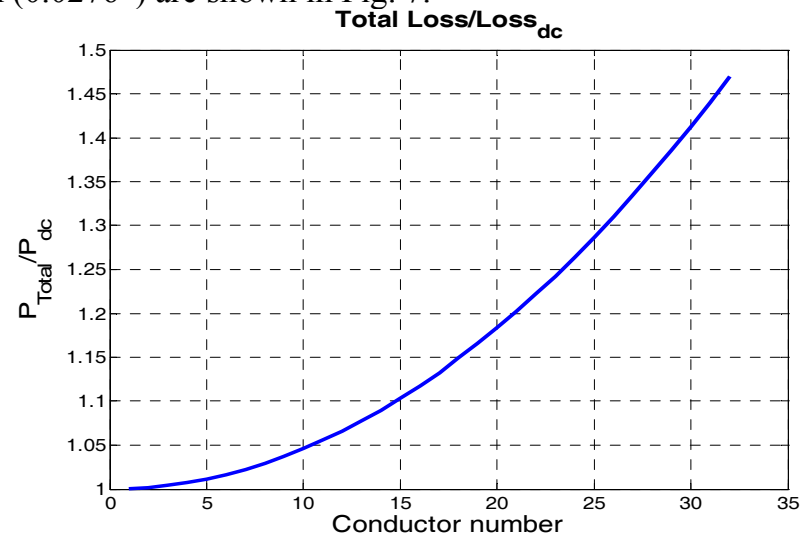

Fig. 7 Typical slot losses for $0.07 \mathrm{~cm}$ thick conductors at $443 \mathrm{~Hz}$ $(0.59 \cdot 15,000$ RPM $)$

The allowed stator current density assuming a 30\% packing factor, a $100^{\circ} \mathrm{C}$ rise, and an ac-dc loss of $1.5: 1$ would be

$J_{\text {stator } @ 30 \% \mathrm{Cu}}=0.3 \cdot \sqrt{\frac{\frac{4.3 \cdot 10^{7}}{1.5} 8.9 \cdot 10^{3} \cdot 383 \cdot 100}{10}}=9.38 \cdot 10^{6} \frac{\mathrm{A}}{\mathrm{m}^{2}}$

The field current $\mathrm{I}_{\mathrm{f}}$ will have the same time dependence as torque since armature current is controlled as constant. Thus, the field current from Fig. 4 can be expressed in terms of its end value $I_{e}$ as

$$
I_{f}=\frac{I_{e} \sqrt{1-\beta}\left(\frac{t}{\tau}\right)}{\sqrt{1-\beta\left(\frac{t}{\tau}\right)^{2}}}
$$

The power dissipation in the rotor conductors with cumulative resistance $R_{f}$ over the excitation time $\tau$ is 


$$
\begin{aligned}
\text { Loss } & =R_{f} \int_{0}^{\tau} I_{f}^{2} d t \\
& =R_{f} \frac{\tau I_{e}^{2}\left(\left\{\tanh ^{-1}(\sqrt{\beta})-\sqrt{\beta}\right\}(1-\beta)\right)}{\beta^{\frac{3}{2}}}
\end{aligned}
$$

Consider a rotor excited with a constant field current $\mathrm{I}_{0}$ for $\tau$ seconds. The dissipation loss in a rotor excited with this current will be identical to the one in the real rotor if

$$
\begin{aligned}
I_{e} & =I_{0} \sqrt{\frac{\beta^{\frac{3}{2}}}{\left(\left\{\tanh ^{-1}(\sqrt{\beta})-\sqrt{\beta}\right\}(1-\beta)\right)}} \\
& =2.198 I_{0}(\text { for } \beta=0.65)
\end{aligned}
$$

Assuming a $100^{\circ}$ change in temperature and a packing fraction of $58 \%$, the ending rotor field current density should be

$$
\begin{aligned}
J_{\text {rotor @ } 58 \% C u} & =\frac{I_{e}}{I_{0}} \cdot 0.58 \cdot \sqrt{\frac{4.3 \cdot 10^{7} \cdot 8.9 \cdot 10^{5} \cdot 383}{10}} \\
& =4.88 \cdot 10^{7} \frac{\mathrm{A}}{\mathrm{m}^{2}}
\end{aligned}
$$

\section{15 MW GENERATOR DESIGN}

When high weight / volume density is a premium, high rotation speed is mandatory. Experience at the Center for Electromechanics shows 15,000 RPM to be a reasonable target. Higher rotation speeds tax available bearings considerably. If the capacitance, charging time, and voltage are specified, the power demand is known from Fig. 2. Optimization is considered by allowing the four parameters in Fig. 8 to vary within a nested loop. The objective is to select the combination of these parameters to minimize weight subject to the constraint the machine deliver the desired torque at speed with the currents constrained by adiabatic heating. The primary difficulty is the torque angle used during this optimization, as subject to be discussed shortly.

Multivariate splines combined with hybrid finite - boundary element are helpful tools for optimizations of this nature. The former allows computation of very complex functions and their derivatives to be performed rapidly, a prerequisite for involved problems with many local minima. The latter allows movement of the rotor and stator without the requirement of re-meshing. The objective function is mapped using multivariate splines to the variables in Fig. 8.

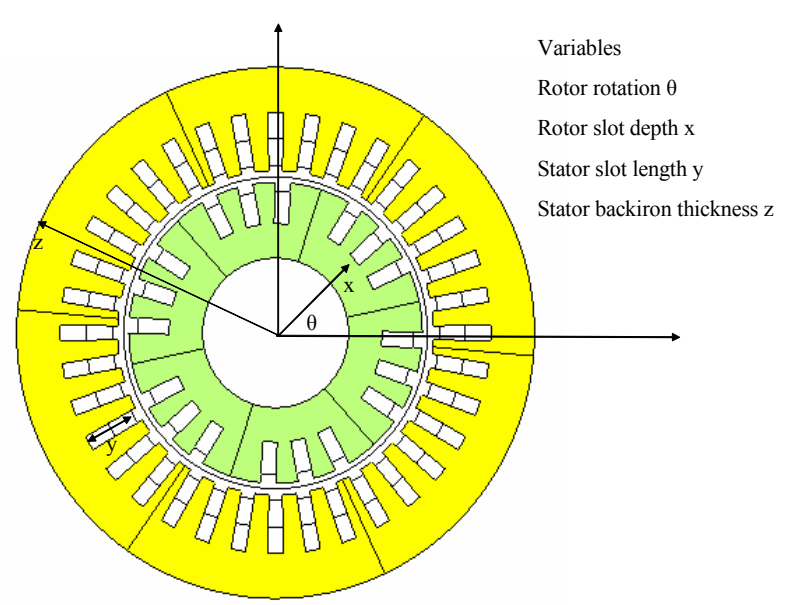

Fig. 8 Optimization Variables.

The four parameters in Fig. 8 are allowed to vary within a nested loop of 384 permutations. Sequential quadratic programming is employed to determine the local minimum using a random starting guess in the variable space examined. The process is repeated multiple times using Monte-Carlo methods to insure the avoidance of local minima. The process is as follows:

1. Construct a nested loop of all unknown parameters. A numerical analysis will be performed on each configuration. The optimization in this study involved 6 steps for the rotor angle position, and four steps for the field slot depth, the stator slot depth, and the stator backiron perturbations, for a total of 384 analyses. Because of the changes considered and the saturation within the steel, adaptive meshing was employed for each analysis. This background computation requires five days of continuous analysis on a Pentium IV, $3 \mathrm{GHz}$ computer, just to get the two dimensional results.

2. After each analysis compute

a. Torque

i. Determine the length of the generator $\mathrm{L}$ required to deliver the power required at the lowest rotation speed., i.e. set the length to be the power required by the rotation speed at time $\tau$.

b. Weight based on slot size, length $\mathrm{L}$, and back iron.

c. Total flux linking each of the stator windings

d. Field and armature current based on slot size

3. After each configuration in step 1, determine the magnetic energy by integrating the product of magnetic vector potential with current. Repeat this calculation with the stator current increased by $\gamma \%$ and with the rotor field current increased by $\gamma \%$. The energy for the machine is known at three excitations. Call these $\mathrm{W}_{0}\left(\mathrm{I}_{\mathrm{s}}, \mathrm{I}_{\mathrm{r}}\right), \mathrm{W}_{1}\left((1+\gamma) \mathrm{I}_{\mathrm{s}}, \mathrm{I}_{\mathrm{r}}\right)$, and $\mathrm{W}_{2}\left(\mathrm{I}_{\mathrm{s}},(1+\gamma) \mathrm{I}_{\mathrm{r}}\right)$, 
each being equal to $\int \vec{A} \cdot \vec{J} d V$. The synchronous

motor is to be thought of as a two terminal system for the purpose of getting the synchronous reactance,

$$
W=\frac{1}{2} L_{s} I_{s}{ }^{2}+\frac{1}{2} L_{r} I_{r}{ }^{2}+M I_{s} I_{r}
$$

When this expression is written three times with the rotor and stator current adjusted appropriately, the synchronous reactance can be computed as

$$
L_{s}=\frac{m}{2} L_{m}=m \frac{W_{1}+W_{2}-2 W_{0}+\gamma\left(W_{1}-W_{0}(3+\gamma)\right)}{I_{s}^{2} \gamma^{2}(2+\gamma)}
$$

4. Use the flux in step 2.c to determine the voltage across the armature winding by multiplying by the mechanical speed, the number of pole pairs, and the turns.

5. Compute the torque angle in terms of the power factor $\mathrm{pf}$ of the load. The conventional vector diagram shown in Fig. 9 is used.

$$
\phi=\tan ^{-1}\left(\frac{I_{a} X_{s} p f}{\frac{V_{L L}}{\sqrt{3}}+I_{a} X_{s} \sqrt{1-p f^{2}}}\right)
$$

Fig. 9 is a phase diagram which shows the relationship of the rotor MMF to the voltage actually induced in the stator winding. The vector diagram is a way to represent the components of the equivalent circuit shown in the lower right inset. $\Phi_{\mathrm{f}}$ is the flux from the rotor. $\mathrm{E}_{\mathrm{f}}$ represents the voltage induced in the stator winding by the rotor field. $\theta$ is the power factor angle dictated by the generator load. Xs is the synchronous reactance of the stator winding, incorporating the coupling to the other phases. The sum of the angles $\theta$ and $\phi$ represents the electrical degrees for which the field winding leads the stator peak torque position. It is also the angle between the rotor MMF and the stator MMF.

6. Use a quad-variate spline to fit the computed quantities for weight and torque angle to the four unknowns shown in Fig. 8 [3].

7. Use a trust region optimization algorithm based on the interior - reflective Newton method to determine the minimum weight [4][5] subject to the constraint that the rotor position angle equal that derived in (17) . The following three points are in order:

a. The length has already been incorporated into the optimization at step 2.a.i.

b. The optimization is dependent on the power factor of the load through step 5 .

c. The real problem has only been computed at 384 separate points. The intermediate space is only approximated using the multivariate spline. It is an approximation demanded by practical considerations.

8. If the working length $\mathrm{L}$ exceeds other restrictions, a second inequality constraint must be added on the maximum length $\mathrm{L}_{\max }$. It is accomplished as follows: a. Torque must be added to weight and torque angle as a third multivariate spline fit in step 6.

b. The requirement on length translates to a requirement on minimum torque as

$$
T_{2 D} \geq \frac{P_{\max }}{\Omega_{\text {final }}}
$$

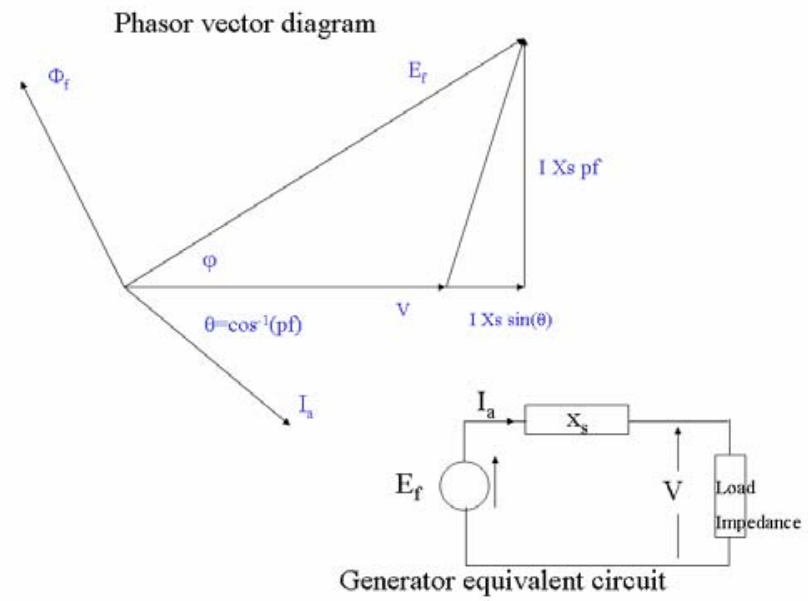

Fig. 9 Vector diagram used to compute the load torque.

\section{A. Optimization Results}

The optimization delivers the following specifications:

36 Stator Slots size $5.8 \mathrm{~cm}$ by $1.63 \mathrm{~cm}$ (2.28" by 0.64 ") centered every 10 degrees

$5 / 6$ pitch winding

Active length $=72.24 \mathrm{~cm}$ (28.44")

Rotor radius $16.56 \mathrm{~cm}$ (6.517")

Inner stator radius $=17.19 \mathrm{~cm}(7.017 ")$

Outer stator radius $=29.21 \mathrm{~cm}\left(11.5^{\prime \prime}\right)$

$15,000 \mathrm{RPM}, 260 \mathrm{~m} / \mathrm{s}$ tip speed

12 Rotor Slots $1.74 \mathrm{~cm}$ by $3.83 \mathrm{~cm}(0.685$ " by 1.41 ") centered every 15 degrees

$\mathrm{Ls}=7.09 \cdot 10^{-5} \mathrm{H}$

$\mathrm{Wt}=1,135 \mathrm{~kg}(2,497 \mathrm{lbs})$

A 0.87 power factor yields a torque angle of 49 degrees. 


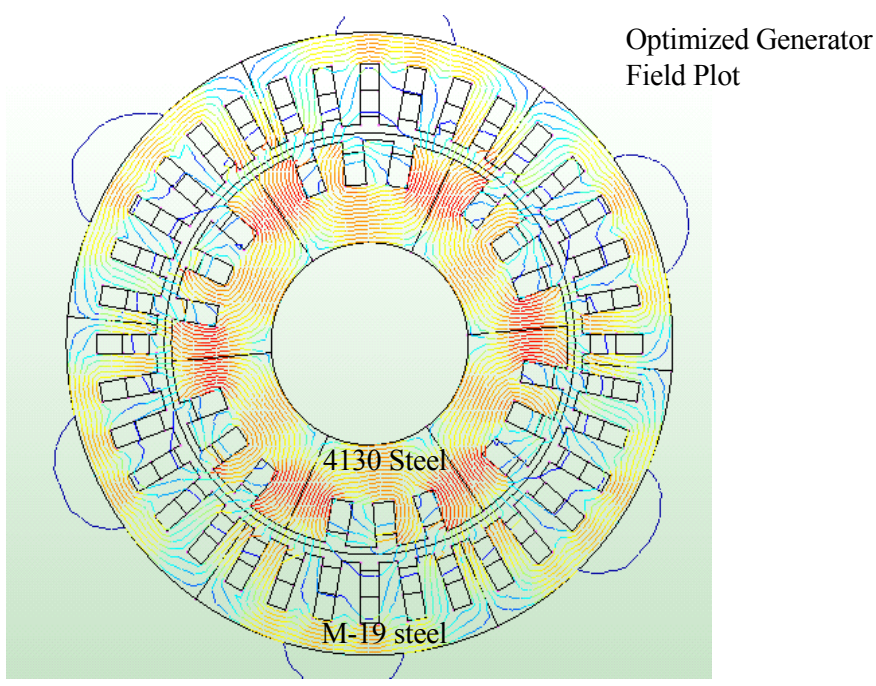

Fig. 10 Optimized stator and rotor layout for $16 \mathrm{MW}$.

The radii breakdown is shown in Fig. 11.

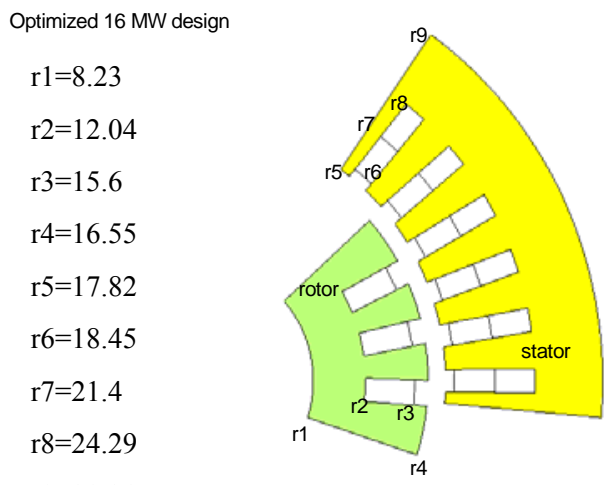

$\mathrm{r} 9=29.21$

Fig. 11 Geometry of the $16 \mathrm{MW}$ generator in $\mathrm{cm}$.

For any given torque angle and slot setting the length required to deliver the required torque at the lowest speed such that the power delivery remains $16 \mathrm{MW}$. With a torque angle of $36^{\circ}$, and stator slot configuration consistent with Fig. 10, the weight as a function of rotor slot depth and stator back iron is shown in Fig. 12.

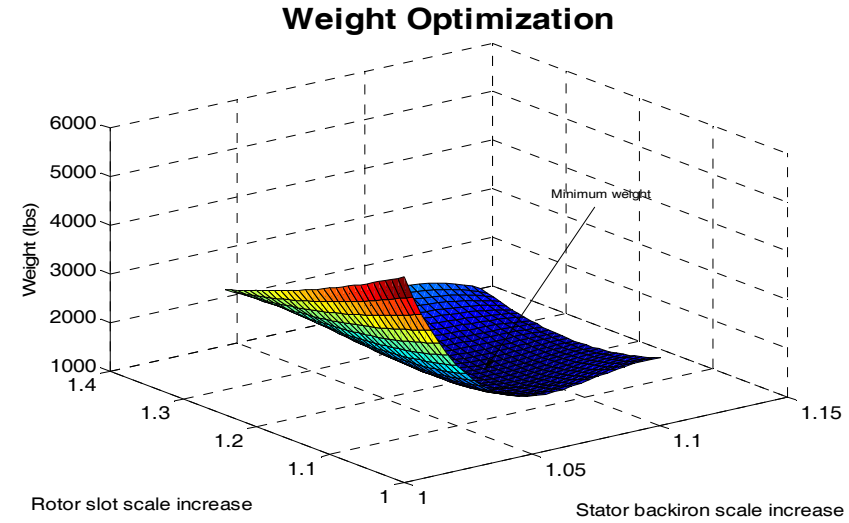

Fig. 12 Weight Optimization for the $16 \mathrm{MW}$ generator.

At each configuration the mid gap B field was computed. Its rms value is shown in Fig. 13. It is clear that the optimization has moved towards the higher $\mathrm{B}$ field region, but not the highest. Too high a weight premium is required for the highest $B$ field regime.

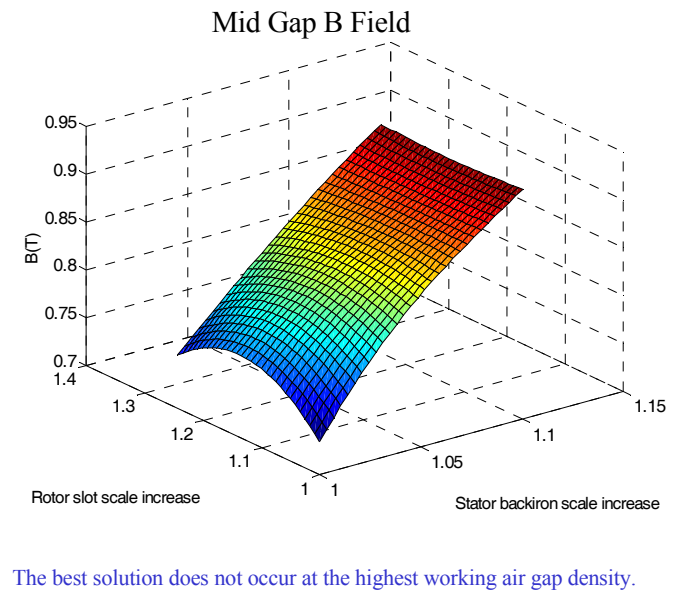

Fig. 13 Mid-gap B field rms value through the optimization.

\section{B. Lamination Losses}

The eddy loss in the laminations is computed by first dropping the conductivity of the steel to a very low value and performing an eddy calculation. The idea is to compute the loss assuming the eddy currents from the bulk steel have a small impact on the primary field components (which they do), and then scale that loss up by the ratio of the real conductivity to the assumed conductivity. If the power dissipated in the rotor per unit length is $\mathrm{P}$,

$$
\text { Energy }_{\text {stator lams }}=\frac{P \cdot L}{\left(L_{\text {/ } \left.\text { Lam }_{\text {thick }}\right)^{2}}^{2}\right.} \cdot 6 \cdot 1.6 \mathrm{~kJ}
$$

Assuming the lamination thickness is $0.47 \mathrm{~mm}(18.5 \mathrm{mil})$, the estimated loss in the stator is $1.12 \mathrm{MJ}$; the stator M-19 has a volume of $94,900 \mathrm{~cm}^{3}\left(5,791 \mathrm{in}^{3}\right)$.

The rotor lamination stack loss is due to the field harmonic content and is estimated to be $170 \mathrm{~kJ}$; the rotor 4130 steel has a volume of $36,953 \mathrm{~cm}^{3}\left(2,255 \mathrm{in}^{3}\right)$. 


\section{CONCLUSIONS}

The choice of current density is completely driven by adiabatic considerations for pulsed short duty cycle electromechanical devices. The choice of appropriate current density for the armature and field windings is determined by adiabatic constraints and is application dependent. Under these conditions the MMF realizable with exciters is considerably larger than that generated with permanent magnets. Once the current density is computed, a self consistent optimization can be completed using parametric analyses.

\section{REFERENCES}

[1] Y. Kawase, T. Yamaguchi, K. Hashimoto, and M. Nakamura, "Effects of Number of Slots in Permanent
Magnets", CEFC - Computational Electromagnetic Fields Conference, Seoul, Korea, June 2004.

[2] Y.Kawase and S.Ito "New Practical Analysis of Electrical Apparatus by 3-D Finite Element Method," Morikita Publishing Co., 2000.

[3] C. de Boor, C., A Practical Guide to Splines, SpringerVerlag, 1978.

[4] Coleman, T.F. and Y. Li, "An Interior, Trust Region Approach for Nonlinear Minimization Subject to Bounds," SIAM Journal on Optimization, Vol. 6, pp. 418-445, 1996.

[5] Coleman, T.F. and Y. Li, "On the Convergence of Reflective Newton Methods for Large-Scale Nonlinear Minimization Subject to Bounds," Mathematical Programming, Vol. 67, Number 2, pp. 189-224, 1994.

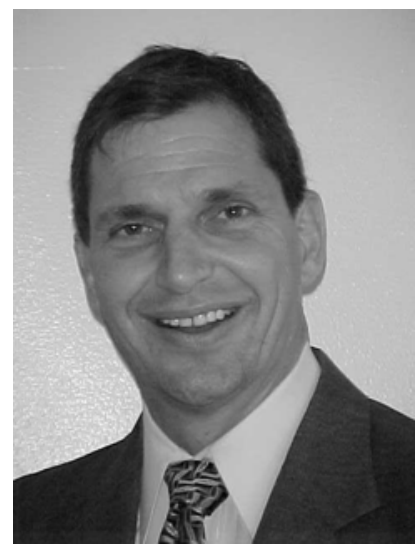

Kent Davey became a Member of IEEE in 1980, a Senior Member in 1986, and a Fellow in 2004. He was born in New Orleans, Louisiana, 1952. $\mathrm{He}$ received hie BS in EE from Tulane in 1974, his MS in Power Engineering from Carnegie Mellon in 1976, and his MS in physics from the University of Pittsburgh in 1976. He received his $\mathrm{PhD}$ from the Massachusetts Institute of Technology in EE in 1980. He completed a Fulbright in Finland on atmospheric physics in 1981.

$\mathrm{He}$ worked at Westinghouse as a large turbine analyzer from 1974 to 1976. From 1979 to 1980 he served as an assistant professor at Texas A\&M University. From 1980 to 1994 he served as a tenured associate professor at the Georgia Institute of Technology. From 1994 to 2002, he served as technical director of American Maglev Technology in Edgewater, Florida. He presently serves as senior research scientist at the Center for Electromechanics at the University of Texas in Austin, Texas. Dr. Davey has research activity in electromechanical machine design, pulsed electromechanical devices, HTS Trapped Field magnets, electroporation, and magnetic stimulation of biological tissue.

Dr. Davey is a Fellow with IEEE, and presently serves as editor of IEEE Transactions on Magnetics. He is actively involved in the review of conference and journal papers for various IEEE activities. 